EUROPEAN ORGANIZATION FOR NUCLEAR RESEARCH

CERN-PPE/93-66

April 20, 1993

\title{
A Search for New Intermediate Vector Bosons and Excited Quarks Decaying to Two-Jets at the CERN $\bar{p} p$ Collider.
}

\section{The UA2 Collaboration}

Bern - Cambridge - CERN - Dortmund - Heidelberg - Melbourne Milano - Orsay (LAL) - Pavia - Perugia - Pisa - Saclay (CEN)

J. Alitti ${ }^{12}$. G. Ambrosini ${ }^{9}$, R. Ansari ${ }^{8}$, D. Autiero ${ }^{11}$, P. Bareyre ${ }^{12}$, I. A. Bertram ${ }^{6}$, G. Blaylock ${ }^{3, a}$, P. Bonamy ${ }^{12}$, K. Borer ${ }^{1}$, M. Bourliaud ${ }^{12}$, D. Buskulic ${ }^{8}$, G. Carboni ${ }^{11}$, D. Cavalli ${ }^{7}$, V. Cavasinni ${ }^{11}$, P. Cenci ${ }^{10}$, J. C. Chollet ${ }^{8}$, C. Conta ${ }^{9}$, G. Costa ${ }^{7}$, F. Costantini ${ }^{11}$, L. Cozzi ${ }^{7}$, A. Cravero ${ }^{7}$, M. Curatolo ${ }^{11}$, A. Dell'Acqua ${ }^{3,9}$, T. DelPrete ${ }^{11}$, R. S. DeWolf ${ }^{2}$, L. DiLella ${ }^{3}$, Y. Ducros ${ }^{12}$, G. F. Egan ${ }^{6}$, K. F. Einsweiler ${ }^{3, b}$, B. Esposito ${ }^{11}$, L. Fayard $^{8}$, A. Federspiel ${ }^{1}$, R. Ferrari ${ }^{9}$, M. Fraternali ${ }^{9, c}$, D. Froidevaux ${ }^{3}$, G. Fumagalli ${ }^{9}$, J. M. Gaillard ${ }^{8,3}$, F. Gianotti ${ }^{7}$, O. Gildemeister ${ }^{3}$, C. Gössling ${ }^{4}$, V. G. Goggi ${ }^{9}$, S. Grünendahl ${ }^{5}$, K. Hara ${ }^{1, c}$, S. Hellman ${ }^{3}$, J. Hřivnáč $\check{c}^{3, d}$, H. Hufnagel ${ }^{4}$, E. Hugentobler ${ }^{1}$, K. Hultqvist ${ }^{3, e}$, E. Iacopini ${ }^{11, f}$, J. Incandela ${ }^{7, g}$, K. Jakobs ${ }^{3, h}$, P. Jenni $^{3}$, E. E. Kluge ${ }^{5}$, N. Kurz ${ }^{5}$, S. Lami ${ }^{11}$, P. Lariccia ${ }^{10}$, M. Lefebvre ${ }^{3, i}$, L. Linssen ${ }^{3}$, M. Livan ${ }^{9}$, P. Lubrano ${ }^{3,10}$, C. Magneville ${ }^{12}$, L. Malgeri ${ }^{j}$, L. Mandelli ${ }^{7}$, L. Mapelli ${ }^{3}$, M. Mazzanti ${ }^{7}$, K. Meier ${ }^{3,5}$, B. Merkel $^{8}$, J. P. Meyer ${ }^{12}$, M. Moniez ${ }^{8}$, R. Moning ${ }^{1}$, M. Morganti1 ${ }^{11}$, L. Müller ${ }^{1}$, D. J. Munday ${ }^{2}$, M. Nessi ${ }^{3}$, F. Nessi-Tedaldi ${ }^{3, k}$, C. Onions ${ }^{3}$, T. Pal ${ }^{1, l}$, M. A. Parker ${ }^{2}$, G. Parrour $^{8}$, F. Pastore ${ }^{9}$, E. Pennacchio ${ }^{9}$, J. M. Pentney ${ }^{3, m}$, M. Pepe ${ }^{3}$, L. Perini ${ }^{7}$, C. Petridou ${ }^{11, n}$, P. Petroff ${ }^{8}$, H. Plothow-Besch ${ }^{3}$, G. Polesello ${ }^{9}$, A. Poppleton ${ }^{3}$, K. Pretzl ${ }^{1}$, M. Primavera ${ }^{11, j}$, M. Punturo ${ }^{10}$, J. P. Repellin ${ }^{8}$, A. Rimoldi ${ }^{9}$, M. Sacchi ${ }^{9}$, P. Scampoli ${ }^{10}$, J. Schacher ${ }^{1}$, B. Schmidt ${ }^{4}$, V. Šimák ${ }^{3, d}$, S. L. Singh ${ }^{2}$, V. Sondermann ${ }^{4}$, R. Spiwoks ${ }^{4}$, S. Stapnes ${ }^{3, o}$, C. Talamonti ${ }^{10}$, F. Tondini ${ }^{10}$, S. N. Tovey ${ }^{6}$, E. Tsesmelis ${ }^{4}$, G. Unal ${ }^{8}$, M. Valdata-Nappi ${ }^{11, j}$, V. Vercesi ${ }^{9}$, A. R. Weidberg ${ }^{3, p}$, P. S. Wells ${ }^{2, q}$, T. O. White ${ }^{2}$, D. R. Wood ${ }^{8, g}$, S. A. Wotton ${ }^{2, q}$, H. Zaccone ${ }^{12}$, A. Zylberstejn ${ }^{12}$

(submitted to Nuclear Physics B - Particle Physics) 


\begin{abstract}
The two-jet invariant mass spectrum as measured in the UA2 experiment is used to search for additional heavy vector bosons decaying to two-jets. The mass of an additional $\mathrm{W}$ boson that couples to fermions with a $V+A$ form is constrained to be greater than $261 \mathrm{GeV}$ to the $90 \%$ confidence level. A limit on the production cross section of additional $\mathrm{W}$ and $\mathrm{Z}$ bosons is given as a function of the boson mass. A limit on the production of excited quarks is also given as a function of the excited quark mass.
\end{abstract}

${ }^{1}$ Laboratorium für Hochenergiephysik, Universität Bern, Sidlerstraße 5, 3012 Bern, Switzerland

${ }^{2}$ Cavendish Laboratory, University of Cambridge, CB3 0HE, UK

${ }^{3}$ CERN, 1211 Geneva 23, Switzerland

${ }^{4}$ Lehrstuhl für Exp. Physik IV, Universität Dortmund, 4600 Dortmund, Germany

${ }^{5}$ Institut für Hochenergiephysik der Universität Heidelberg, Schröderstraße 90, 6900 Heidelberg, Germany

${ }^{6}$ School of Physics, University of Melbourne, Parkville 3052, Australia

${ }^{7}$ Dipartimento di Fisica dell'Università di Milano and Sezione INFN Milano, 20133 Milano, Italy

${ }^{8}$ Laboratoire de l'Accélérateur Linéaire, Université de Paris-Sud, 91405 Orsay, France

${ }^{9}$ Dipartimento di Fisica Nucleare e Teorica, Università di Pavia and INFN, Sezione di Pavia, Via Bassi 6, 27100 Pavia, Italy

${ }^{10}$ Dipartimento di Fisica dell'Università di Perugia and INFN, Sezione di Perugia, Via Pascoli, 06100 Perugia, Italy

${ }^{11}$ Dipartimento di Fisica dell'Università di Pisa and INFN Sezione di Pisa, Via Livornese, S. Piero a Grado, 56100 Pisa, Italy

${ }^{12}$ Centre d'Etudes Nucléaires de Saclay, 91191 Gif-sur-Yvette Cedex, France

a) Now at University of California, Santa Cruz, USA

b) Now at Lawrence Berkeley Laboratory, Berkeley, California, USA

c) Now at University of Tsukuba, Tsukuba, Ibaraki 305, Japan

d) Now at Institute of Physics, CSAV, Praha 8, Czechoslovakia

e) Now at University of Stockholm, Stockholm, Sweden

f) Also at Scuola Normale Superiore, Pisa, Italy

g) Now at Fermi National Accelerator Laboratory, Batavia, Illinois, USA

h) Now at Max Plank Institut für Physik, D 8000 München, Germany

i) Now at University of Victoria, Victoria, Canada

j) Now at Dipartimento di Fisica dell'Università della Calabria e gruppo INF N, Cosenza, Italy

k) Now at Eidgenössische Technische Hochschule, ETH Zürich, CH 8093 Zürich, Switzerland

l) Now at SSCL, 2550 Beckleymeade Av., Dallas, Texas, USA

m) Now at Department of Physics, Brunel University, Uxbridge, London, UK

n) Now at INF N Sezione di Trieste, Laboratori Area di Ricerca, Padriciano 99, 34012 Trieste, Italy

o) Now at Physics Institute, University of Oslo, Blindern, Oslo, Norway

p) Now at Nuclear Physics Laboratory, University of Oxford, Oxford, UK

q) Now at CERN, Geneva, Switzerland 


\section{Introduction}

The predictions of the Standard Model of particle physics have been confirmed by current high energy experiments with no significant observed deviations from theory.

One of the proposed extensions of the Standard Model is the existence of new Heavy Vector Bosons (HVB) that couple to fermions with a $V+A$ form. If leptonic decays of a right handed $\mathrm{W}, W_{R}$, are suppressed by a heavy or non existent right handed neutrino then hadronic decay modes will be the only way of experimentally observing the decays of the $W_{R}$. This search will also examine the possible existence of an additional $Z$ boson, $Z^{\prime}$, and of excited quarks. Searches for HVB in the leptonic decay channels have so far produced the best direct mass bounds for these particles [1].

The production of hadronic jets is the dominant contribution to high transverse momentum $\left(p_{T}\right)$ processes in proton-antiproton $(\bar{p} p)$ collisions. High $p_{T}$ jets have been observed since the early phase of experimentation at the CERN $\bar{p} p$ collider [2] and their production properties are well described by perturbative QCD [3].

The $W_{R}$ and $Z^{\prime}$ are searched for via their two-jet decay channels, $W_{R} \rightarrow \bar{q} q^{\prime}$ and $Z^{\prime} \rightarrow \bar{q} q$. Since hadronic jets produced in strong interactions and those produced in the decays of additional HVB are indistinguishable on an event-by-event basis, the overall mass spectrum produced by $\bar{p} p$ interactions has to be examined.

A previous search [4] for $\mathrm{W}$ and $\mathrm{Z}$ bosons in the two-jet mass spectrum revealed a signal of $5618 \pm 1334$ events with shape and position consistent with expectations. In that analysis a search for the existence of the $W_{R}$ excluded its existence to a $90 \%$ confidence level for the mass range of $101 \mathrm{GeV}<M<158 \mathrm{GeV}$. The analysis was based on a data sample corresponding to an integrated luminosity of $4.6 \mathrm{pb}^{-1}$ collected in 1989. The present analysis uses data collected during the 1989 and 1990 collider runs, corresponding to a data sample of $10.9 \mathrm{pb}^{-1}$.

The following section briefly describes the components of the UA2 detector relevant to this analysis. Section 3 explains the jet identification and reconstruction methods used at the trigger level and in the data analysis. The two-jet mass spectrum is described in section 4 . In section 5 the search for additional HVB is described and mass limits for the existence of a $W_{R}$ and a $Z^{\prime}$ are presented. The results from a search for excited quarks are also briefly reported.

\section{The UA2 Apparatus}

A description of the UA2 apparatus can be found in Ref [5]. In order for the UA2 trigger and data acquisition system to handle the high jet production rates with minimal dead-time, data from tracking detectors were not read out for low mass 
two-jet candidates.

\subsection{Calorimetry}

The main features of the UA2 calorimeter for this analysis will be summarised here. A more complete description can be found in Ref [6].

Calorimetry is provided over the full azimuthal range, $0^{\circ}<\phi<360^{\circ}$, and the pseudorapidity region $|\eta|<3$. The calorimeters are subdivided into the Central Calorimeter (CC) covering the pseudorapidities $|\eta|<1$, and two end cap calorimeters (EC) covering the regions $0.9<|\eta|<3$.

The CC is segmented into 240 cells subtending $10^{\circ}$ in $\theta$ and $15^{\circ}$ in $\phi$. The electromagnetic part of the calorimeter is a multilayer sandwich of lead and scintillator 17 radiation lengths deep, while the hadronic part, subdivided into two compartments, is an iron-scintillator sandwich, resulting in a depth of 4.5 hadronic absorption lengths, including the electromagnetic compartment.

Each of the ECs consists of 12 azimuthal modules, each subdivided into 16 cells. While the cells closest to the beam axis $(2.5<|\eta|<3.0$ and $2.2<|\eta|<2.5)$ cover $30^{\circ}$ in azimuth, all cells in the interval $1.0<|\eta|<2.5$ have a segmentation of $\Delta \phi=15^{\circ}$ and $\Delta \eta=0.2$, with one electromagnetic and one hadronic compartment. The electromagnetic compartment is a multi-layer sandwich of lead and scintillator with a total thickness varying from 17.1 to 24.4 radiation lengths depending on the polar angle. The hadronic calorimeter is a multi-layer sandwich of iron and scintillator corresponding to about 6.5 absorption lengths, including the electromagnetic cells. Cells with only hadronic calorimetry cover the pseudorapidity range $0.9<|\eta|<1.0$ to measure the energy of particles which would otherwise escape detection in the interface between the $\mathrm{CC}$ and the $\mathrm{EC}$ modules. To minimize dead space in the boundaries between two neighbouring EC modules, the modules have been rotated by $50 \mathrm{mr}$ around their symmetry axis normal to the beam.

The initial absolute calibration of the calorimeters was obtained by exposing every cell to beams of electrons, pions and muons of known momenta. The calibration stability was monitored by measuring the calorimeter response to a radioactive source $\left(C o^{60}\right)$. The accuracy of this relative response monitoring has been measured by periodic recalibrations of a part of the calorimeter using a test beam. The uncertainty on the absolute calibration was determined to be $\pm 1 \%$ for the electromagnetic energy scale and $\pm 2 \%$ for the hadronic energy scale. Since hadronic jets typically deposit one half of their energy in the electromagnetic compartment, the uncertainty on the absolute jet energy scale is $\pm 1.5 \%$.

The response of the UA2 calorimeter to electrons is different from that to hadrons and in particular to pions. Weight factors are defined for all the compartments of the 
calorimeter to compensate for this difference. The software weights applied to the electromagnetic cells are 1.18 in the $\mathrm{CC}$ and 1.2 in the EC. An additional software weight of 1.06 is applied to the second hadronic compartment of the $\mathrm{CC}$ to account for hadronic energy leaking out the back of the calorimeter.

This procedure ensures the correct energy measurement for particles with momenta equal to those of the calibration beam. Since a jet fragment typically carries a small fraction of the parent partons momentum, jet energy measurements are sensitive to deviations from calorimeter linearity at low momenta. Test beam measurements performed with the EC showed non-linearities at very low hadron momenta $(<1 \mathrm{GeV})$. For example, only $70 \%$ of the total energy of a charged pion with a momentum of $200 \mathrm{MeV} / \mathrm{c}$ is measured by the calorimeter.

\subsection{Time-of-Flight Detector (TOF)}

Two arrays of 20 scintillation counters at a distance of $\pm 120 \mathrm{~cm}$ from the centre of the collision region cover the pseudorapidity region $2.3<|\eta|<4$.1. The layout is described in Ref [7]. For this analysis these detectors serve two purposes:

1. In the absence of track reconstruction, the event vertex is determined by measuring the time-of-flight difference between the particles emitted along the $p$ and $\bar{p}$ beams. Averaging over the counter surface, a time resolution of $300 \mathrm{ps}$ has been achieved, providing a determination of the event vertex along the beam with a r.m.s. precision of $45 \mathrm{~mm}$.

2. At the trigger level, the detector selects inelastic $\bar{p} p$ interactions by demanding a coincidence between particles detected in the two arrays at the time of nominal beam crossing. This coincidence defines a beam-beam interaction signal.

\subsection{Luminosity}

The luminosity is measured using eight scintillator telescopes at small angles to the beam, four on each side of the detector. These telescopes consist of pairs of scintillation counters $8 \mathrm{~m}$ and $10 \mathrm{~m}$ from the interaction point. Each pair of counters is read out in timing coincidence, sensitive only to particles travelling outwards from the interaction region at the time of nominal beam crossing. The accumulated rates of these coincidences are used to compute the integrated luminosity using an algorithm which accounts for the effect of multiple interactions.

The data sample for this analysis corresponds to an integrated luminosity of $10.9 \pm 0.6 p b^{-1}$, where the uncertainty is dominated by a $4.7 \%$ uncertainty in the 
knowledge of the total cross-section.

\section{Jet Identification}

A two-level trigger system is used, based on calorimeter information and signals from the TOF detector.

The large QCD two-jet cross section has imposed a trigger strategy based on two different mass thresholds. A "low mass trigger" was used to collect data with the full integrated luminosity. In 1989 this trigger accepted events with a reconstructed mass exceeding $66 \mathrm{GeV}$ whilst in 1990 the trigger accepted events with a mass exceeding $80 \mathrm{GeV}$. In 1989 the mass region down to $44 \mathrm{GeV}$ was also sampled by a "very low mass trigger" which was pre-scaled by a factor 8 so that a sample corresponding to an integrated luminosity of $0.56 \mathrm{pb}^{-1}$ was collected.

The angular distribution of jets from decays of heavy vector bosons is expected to be less peaked in the forward direction than that of the QCD processes dominated

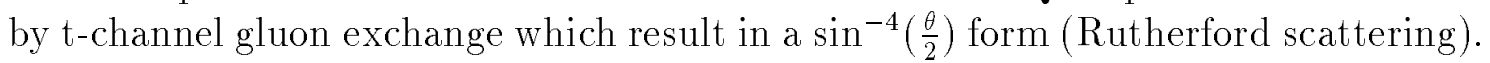
For this reason the trigger uses only information from the $\mathrm{CC}$ because of a more favourable signal to background ratio.

\subsection{Jet Identification at the Trigger Level}

The jets selected by the first level trigger are measured in $90^{\circ}$ wide azimuthal wedges covering the pseudorapidity range of the $\mathrm{CC}$. A coincidence is required between two such wedges at opposite azimuth, each with an energy deposition exceeding a threshold set typically at values between 17 and $25 \mathrm{GeV}$.

The second level trigger refines the crude jet identification that is implemented in the first level trigger. At this stage the $E_{T}$ of each of the cells is available in digitised form with individual calibration constants applied. After finding the leading $90^{\circ}$ wedge, the leading $7 \times 5\left(\Delta \theta \times \Delta \phi=70^{\circ} \times 75^{\circ}\right)$ rectangular window within this wedge is found and compared with the $E_{T}$ threshold. All $7 \times 5$ rectangular windows that are back-to-back to within $30^{\circ}$ in $\phi$ of the leading window are then constructed. It is required that at least one of these windows has an $E_{T}$ deposition greater than the threshold. The size of the fixed jet aperture has been optimised from measurements of the transverse energy flow in two-jet events. For the "low mass trigger" the $E_{T}$ threshold is typically set at values between 13 and $22 \mathrm{GeV}$. The threshold of the 1989 "very low mass trigger" is $10 \mathrm{GeV}$. 


\subsection{Final Jet Identification and Measurement}

The data that are written to tape after passing the two trigger levels represent a sample of genuine two-jet events. The jet algorithm is optimised in order to achieve the best possible energy resolution.

Before applying the refined jet algorithm, the pseudorapidity of each of the jets is calculated using the vertex position along the beam axis as measured by the TOF detector. The three leading $7 \times 5$ windows are found for the pseudorapidity range $|\eta|<2.0$. This is done to ensure that there is no significant jet activity in the endcap calorimeters and that the events are in fact two-jet events and not multi-jet events. The rectangular shape of the windows does not accurately reflect the conical symmetry of hadronic jets. Therefore, the jet energy is remeasured by summing the $E_{T}$ in a cone with a circular cross section in the $\eta, \phi$ plane, which reflects the symmetry of a typical jet. The direction of the jet is given by a vector pointing from the TOF vertex to the centre of the calorimeter cell with the maximal $E_{T}$ deposition (this vector defines the cone axis of the jet).

The cone size affects the jet energy resolution in two ways. If the cone is too small then not all of the jets energy is contained in the cone and the jet energy is underestimated. If the cone size is too large then particles that are not part of the jet are included in the jet, leading to an overestimate of the jet energy. These two effects are balanced to optimise the two-jet mass reconstruction. Based on studies presented in Ref [4], the cone size used to measure the jet energies in this analysis is $\left(\Delta \eta^{2}+\Delta \phi^{2}\right)<0.64$. Conservatively, electrons and photons have not been explicitly removed from the data sample.

\section{Two Jet Mass Spectrum}

\subsection{Analysis Cuts}

A series of cuts are applied to the data sample that passes the second level trigger to remove remaining multi-jet events. After ordering the jets in each event according to decreasing transverse energy $\left(E_{T}^{1}, E_{T}^{2}, \ldots\right)$ the following set of cuts are applied.

1. Vertex Cut

A cut on the $z$ position of the vertex as measured by the TOF detector is made to ensure that all the events occur well inside the detector. Only events in the range

$$
-200 \mathrm{~mm}<z<200 \mathrm{~mm}
$$

are accepted in the final sample.

2. Polar Angle Cut

A cut on the polar angles $\theta$ of the two leading jets is made to ensure that 
the two leading jets are well contained in the $\mathrm{CC}$. The two leading jets are required to satisfy $|\cos \theta|<0.6$.

\section{Cut on Additional Jet Activity}

The cut

$$
E_{T}^{3}<30 G e V
$$

is applied to ensure an exclusive two-jet sample by vetoing on additional jet activity.

These cuts reject $60.8 \%$ of the events that pass the two-jet "low mass trigger" leaving approximately $3.5 \times 10^{5}$ events with $M_{J J}>80 \mathrm{GeV}$ in the final sample.

\subsection{Efficiency of the Analysis Cuts}

The efficiencies of the two-jet trigger and of the analysis cuts are calculated using a sample of $Z^{\prime}$ events produced using the event generator of Ref [8] and a simulation of the UA2 detector. The event generator has been tuned to account for the effect of underlying events, and of pile-up whose rate depends on the collider's luminosity. The fragmentation of the partons is simulated using the standard prescription of Ref [9], which uses the string fragmentation scheme. The analysis cuts are applied to the Monte Carlo data and the following efficiencies are calculated:

\section{Trigger Efficiency}

The trigger efficiency, $\epsilon_{T R I G}$, is given by the fraction of Monte Carlo events that pass the trigger requirements.

\section{TOF Efficiency}

This is the fraction of events with good TOF information, $\epsilon_{T O F}=98 \pm 1 \%$, as determined from data.

\section{Vertex Cut Efficiency}

The Vertex Cut efficiency, $\epsilon_{V T X}$, is defined by the percentage of events that pass the vertex cut. This efficiency is calculated using the data and is found to be $88.9 \pm 0.1 \%$.

\section{Central Calorimeter Acceptance}

The $\mathrm{CC}$ acceptance, $\epsilon_{C C}$, is defined as the fraction of events that pass the trigger and the vertex cut and have the two leading jets contained within the central calorimeter.

\section{Efficiency of the Cut on Third Jet}

This efficiency, $\epsilon_{E_{T}^{3}}$, is the percentage of legitimate two-jet events that pass the cut on additional jet activity. 
The overall efficiency of these cuts is given by the product:

$$
\epsilon_{C U T S}=\epsilon_{T R I G} \cdot \epsilon_{T O F} \cdot \epsilon_{V T X} \cdot \epsilon_{C C} \cdot \epsilon_{E_{T}^{3}}
$$

These efficiencies are calculated for various $Z^{\prime}$ masses and with the correct mixture of underlying events for the combined data taking periods. The results of these calculations are given in Table 1.

Table 1: Efficiencies of trigger and analysis cuts for $Z^{\prime} \rightarrow \bar{q} q$ calculated using Monte Carlo data with an underlying event mixture corresponding to the combined data taking periods (all results are given in percentages).

\begin{tabular}{|c|c|c|c|c|c|c|}
\hline$M_{Z^{\prime}}$ & $100 \mathrm{GeV}$ & $150 \mathrm{GeV}$ & $200 \mathrm{GeV}$ & $250 \mathrm{GeV}$ & $300 \mathrm{GeV}$ & $350 \mathrm{GeV}$ \\
\hline$\epsilon_{T R I G}$ & $38.8 \pm 0.7$ & $49.3 \pm 0.9$ & $55.7 \pm 0.9$ & $58.4 \pm 1.0$ & $59.5 \pm 1.0$ & $58.8 \pm 1.0$ \\
\hline$\epsilon_{C C}$ & $53.6 \pm 1.5$ & $55.1 \pm 1.3$ & $57.1 \pm 1.3$ & $60.1 \pm 1.3$ & $61.0 \pm 1.3$ & $63.7 \pm 1.3$ \\
\hline$\epsilon_{E_{T}^{3}}$ & $99.6 \pm 3.1$ & $98.6 \pm 2.7$ & $97.4 \pm 2.5$ & $95.6 \pm 2.3$ & $95.2 \pm 2.3$ & $94.9 \pm 2.3$ \\
\hline \hline$\epsilon_{C U T S}$ & $18.0 \pm 0.8$ & $23.4 \pm 1.0$ & $27.0 \pm 1.0$ & $29.2 \pm 1.1$ & $30.1 \pm 1.1$ & $30.9 \pm 1.1$ \\
\hline
\end{tabular}

\subsection{The Combined Data Sample}

The data collected during the two data-taking periods have to be combined. The 1989 two-jet mass spectrum has a threshold of $M_{J J}>48 \mathrm{GeV}$ for full efficiency, whilst the 1990 two-jet mass spectrum has a threshold of $M_{J J}>110 \mathrm{GeV}$ because of the higher trigger thresholds. The two data samples are merged by adjusting the 1990 two-jet mass scale to that of the 1989 by adding a scale correction of $\Delta M_{90}=-2.0 \mathrm{GeV}$. This correction is required by changes in the proportion of underlying events and by calibration uncertainties of the 1990 two-jet mass spectrum. Below $110 \mathrm{GeV}$ only the 1989 data is used; above $110 \mathrm{GeV}$ the data samples are merged.

\section{Search for New Particles Decaying to Two-Jets}

\subsection{Fitting Procedure}

The method used to search for new particles decaying into two-jets is outlined in this section.

Before searching for a signal from the decay $X \rightarrow \bar{q} q^{\prime}$ the QCD two-jet background and the signal from $W \rightarrow \bar{q} q^{\prime}$ and $Z \rightarrow \bar{q} q$ have to be fitted. All fits have 
been performed over the two jet mass range $48<M_{J J}<300 \mathrm{GeV}$. The QCD two-jet background is described by the following parametrisation [4]:

$$
B(\alpha, \beta, \gamma)=M_{J J}^{-\alpha} e^{-\beta M_{J J}} e^{-\gamma M_{J J}^{2}}
$$

The signal from the two-jet decays of the $\mathrm{W}$ and $\mathrm{Z}$ bosons is described as follows [4]:

- The mass resolution $\sigma_{W}$ of the calorimeter for the decay $W \rightarrow \bar{q} q^{\prime}$ is $10.7 \%$, which is much larger than the natural width of the W. The experimentally observed line shape is therefore approximated by a Gaussian form and the measured cross-section is corrected to take into account the effect of nonGaussian tails.

- The $W$ and $Z$ mass peaks in the two-jet mass spectrum are expected to be shifted by instrumental effects like calorimeter non-linearities, particle losses outside the jet cone, etc. However, the mass ratio between the $\mathrm{W}$ and the $\mathrm{Z}$ is not affected by the systematic error on the mass scale. All fits assume a fixed mass ratio $\mathrm{r}=M_{Z} / M_{W}=1.135$ based on a recent measurement of the decays $W \rightarrow e \nu$ and $Z \rightarrow e^{+} e^{-}$using the same calorimeter [10].

- The relative contributions from the $\mathrm{W}$ and $\mathrm{Z}$ decays to the observed peak structure is given by the Standard Model predictions after correcting for the relative detection efficiencies. Taking the experimentally observed cross-section ratio of $W, Z$ production in the $Z \rightarrow e^{+} e^{-}$and $W \rightarrow e \nu$ decay modes [11], the Standard Model ratios of the $\mathrm{W}$ and $\mathrm{Z}$ partial widths into quarks and leptons (assuming five quark flavours) and the relative detection efficiencies [4] for $W \rightarrow \bar{q} q^{\prime}$ and $Z \rightarrow \bar{q} q$ decays, a ratio $C=\sigma(Z \rightarrow \bar{q} q) / \sigma\left(W \rightarrow \bar{q} q^{\prime}\right)=0.341$ has been used.

Under these assumptions the line shape of the $\mathrm{W}$ and $\mathrm{Z}$ decays to two-jets is described by the following double Gaussian

$$
S_{W, Z}\left(M_{W}, \sigma_{W}, N_{W}\right)=\frac{N_{W}}{\sqrt{2 \pi} \sigma_{W}} e^{-\frac{1}{2}\left(\frac{M-M_{W}}{\sigma_{W}}\right)^{2}}+\frac{N_{W} C}{\sqrt{2 \pi} r \sigma_{W}} e^{-\frac{1}{2}\left(\frac{M-r M_{W}}{r \sigma_{W}}\right)^{2}}
$$

where $N_{W}(1+C)$ is the total number of $\mathrm{W}$ and $\mathrm{Z}$ decays to two-jet final states.

Finally, the two-jet decay of a hypothetical particle $\mathrm{X}$ is represented by a single Gaussian:

$$
S_{X}\left(N_{X}, M_{X}, \sigma\right)=\frac{N_{X}}{\sqrt{2 \pi} \sigma} e^{-\frac{1}{2}\left(\frac{M-M_{X}}{\sigma}\right)^{2}}
$$

where $\sigma$ describes the experimental width of the decay peak at mass $M_{X}$, as obtained from the natural width of the particle, $\Gamma_{X}$, after convolution with the mass resolution of the calorimeter. The overall fit function (with an overall normalisation factor $f$ ) is then

$$
G=f\left[B(\alpha, \beta, \gamma)+S_{W, Z}\left(M_{W}, \sigma_{W}, N_{W}\right)+S_{X}\left(N_{X}, M_{X}, \sigma\right)\right]
$$


and the best fit is obtained by minimizing the $\chi^{2}$ value, defined as

$$
\chi^{2}=\begin{array}{ll}
\sum_{i}\left(\frac{G_{i}-D_{i}}{\sqrt{D_{i}}}\right)^{2} & \left(D_{i}>0\right) \\
\sum_{i}\left(\frac{G_{i}}{\sqrt{G_{i}}}\right)^{2} & \left(D_{i}=0\right)
\end{array}
$$

where $G_{i}$ is the value of the fit function in each of the histogram bins and $D_{i}$ is the number of events in each histogram bin (bin size $2 \mathrm{GeV}$ ).

In this fit the mass and resolution of the $\mathrm{W}$ and $\mathrm{Z}$ peak are fixed to the values found by the fit to the data spectrum. The fit is carried out for free $M_{X}$ and $\sigma$ and no evidence is found for the decay $X \rightarrow \bar{q} q^{\prime}$. Hence mass limits for various non-standard model particles decaying to two-jets are determined.

This is done by fixing the mass $M_{X}$ of the particle X, fixing its natural width to that predicted by theory and then fitting the data. The width of the decay peak, $\sigma$, is determined by Monte Carlo studies as described below. This measurement is then repeated for a series of values of $M_{X}$ and the experimental cross sections for all of these measurements is calculated from the equation:

$$
\sigma_{X}\left(M_{X}\right)=\frac{N_{X}}{\epsilon_{X}\left(M_{X}\right) \mathcal{L}}
$$

where $N_{X}$ is the number of decays $X \rightarrow \bar{q} q^{\prime}$ found by the fitting process at mass $M_{X}, \mathcal{L}$ is the integrated luminosity of the sample and $\epsilon_{X}\left(M_{X}\right)$ is the efficiency of finding these decays at $M_{X}$.

From the value of the experimental cross section so obtained, one can calculate the $90 \%$ confidence limits (CL), defined as the value of the cross-section corresponding to $90 \%$ of the physical region of the probability distribution function given by the experimental cross section and its error, $\sigma_{X} \pm \Delta \sigma_{X}$.

The systematic uncertainties of the fitting method are estimated by making nine independent fits. The uncertainty in the decay peak width, $\sigma$, is accounted for by repeating the fitting procedure at each value of $M_{X}$ with the decay width given by $\sigma$, $\sigma+\Delta \sigma$ and $\sigma-\Delta \sigma$. The uncertainty in the mass shift, $\Delta M_{90}$, required to combine the 1989 and 1990 data sets, is accounted for by repeating the above set of fits for $\Delta M_{90}=-2.0 \pm 0.5 \mathrm{GeV}$.

\subsection{Monte Carlo Studies of the Fitting Program}

The values of $\sigma, \epsilon_{X}$ and the "mass response" used in this analysis are determined by a Monte Carlo simulation which involves producing a background spectrum including QCD interactions and W,Z decays to two-jets. Monte Carlo generated $X \rightarrow \bar{q} q^{\prime}$ 
Table 2: Mass Response, R, $\sigma$ and efficiency of the fitting procedure.

\begin{tabular}{|c|c|c|c|c|c|}
\hline $\begin{array}{c}M_{X} \\
(\mathrm{GeV})\end{array}$ & $\begin{array}{c}\Gamma_{X} \\
(\mathrm{GeV})\end{array}$ & $\begin{array}{c}\mathrm{R} \\
\left(M_{J J} / M_{X}\right)\end{array}$ & $\begin{array}{c}\sigma \\
(\%)\end{array}$ & $\begin{array}{c}\epsilon_{f i t} \\
(\%)\end{array}$ & $\begin{array}{c}\epsilon_{A L L} \\
(\%)\end{array}$ \\
\hline 150 & 4.5 & $0.94 \pm 0.01$ & $9.0 \pm 1.2$ & $68.3 \pm 7.2$ & $16.0 \pm 1.8$ \\
200 & 6.0 & $0.954 \pm 0.004$ & $9.1 \pm 1.1$ & $69.7 \pm 10.4$ & $18.8 \pm 2.9$ \\
250 & 7.5 & $0.954 \pm 0.003$ & $7.8 \pm 0.2$ & $70.6 \pm 12.0$ & $20.6 \pm 3.6$ \\
300 & 8.9 & $0.959 \pm 0.002$ & $7.9 \pm 0.2$ & $69.2 \pm 9.9$ & $20.8 \pm 3.1$ \\
350 & 10.4 & $0.949 \pm 0.002$ & $8.8 \pm 0.2$ & $68.7 \pm 3.4$ & $21.2 \pm 3.4$ \\
\hline
\end{tabular}

events with a natural width $\Gamma_{X}$ are added to this background and the whole spectrum is fitted. First the Monte Carlo mass spectrum is fitted with free values for $\sigma$ and $M_{X}$ to determine the peak width and mass response, $R=M_{J J} / M_{X}$ where $M_{J J}$ is the position of the peak of the Gaussian fit. The mass, $M_{X}$, and the width of the decay peak, $\sigma$, are then fixed to the values determined by the above procedure. The fits are repeated to determine the efficiency of the fitting procedure and hence the overall efficiency of detecting $X \rightarrow \bar{q} q^{\prime}$ decays. The results of these simulations are given in Table 2.

These results show that the fitting efficiency, $\epsilon_{f i t}$, is mass independent and is approximately $70 \%$. The main cause for the inefficiency is the low mass tail present in the measured $X$ mass distribution (see Fig 1 ). This tail is reabsorbed in the parametrisation of the QCD background and is not included in the signal found by the fitting function.

\subsection{Mass Limits for a Right Handed W Boson}

The first particle to be discussed is an additional intermediate vector boson, the right handed $\mathrm{W}$ boson, $W_{R}$, which couples to fermions with a $\mathrm{V}+\mathrm{A}$ form. The model examined here assumes that all couplings have the same strength as in the Standard Model. If the $W_{R}$ exists and the right-handed neutrino either does not exist or has a very high mass, then only the two-jet decay channel is open. Under the assumption that the decay $W_{R} \rightarrow t \bar{b}$ is forbidden because the mass of the top quark is too large, then the natural width for the $W_{R}, \Gamma_{W_{R}}$, is given by the width of the $\mathrm{W}$ boson scaled by the mass ratio

$$
\Gamma_{W_{R}}=\frac{2}{3}\left(\frac{M_{W_{R}}}{M_{W}}\right) \Gamma_{W}
$$

where the factor of $\frac{2}{3}$ accounts for the absence of the leptonic decays. The mass of the $W$ and its width are taken from Ref [12]. 


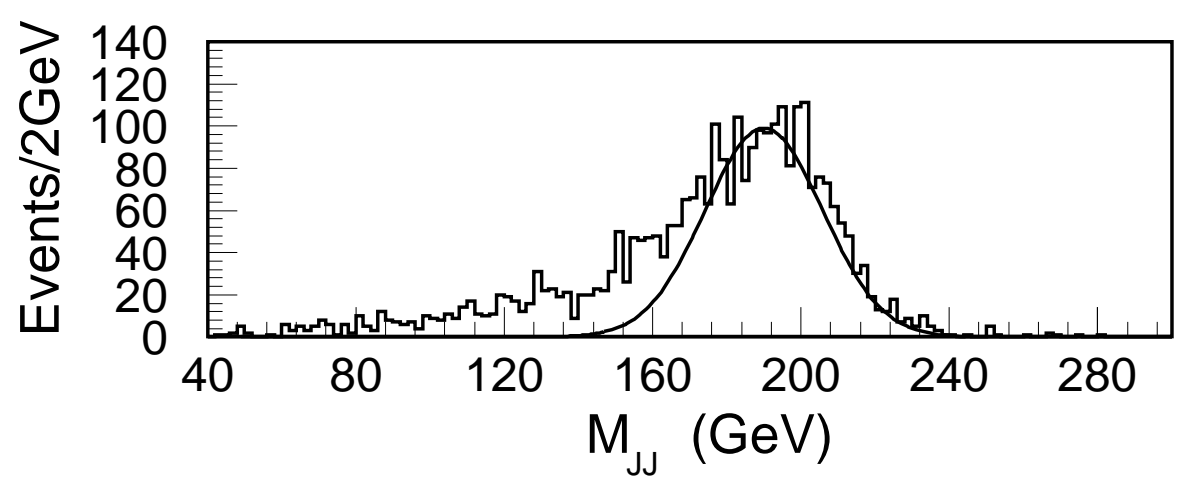

Figure 1: Monte Carlo generated $Z^{\prime}$ of mass $200 \mathrm{GeV}$ decaying to two-jets. The curve shows the best fit to the signal.

The mass value obtained from the fit must then be corrected to give the true value of $M_{W_{R}}$. This is done by dividing $M_{X}$ by the mass response less its error

$$
M_{W_{R}}=\left(\frac{M_{X}}{R\left(M_{X}\right)-\Delta R\left(M_{X}\right)}\right) .
$$

This produces conservative mass limits.

The result of the nine separate fits carried out as described at the beginning of section 5 are shown in Fig 2. The overall $90 \%$ confidence limits is then determined as the largest of the $90 \%$ CL for each mass $M_{X}$. This limit is then compared with a $W_{R}$ production cross section calculated assuming that it has the same couplings as the $\mathrm{W}$ boson. The cross section is obtained from a calculation developed by [13], using the structure function set from Ref [14] with $\Lambda_{Q C D}=260 \mathrm{MeV}$, which is chosen because it gives the smallest cross section value whilst being consistent with the measured value of $\Lambda_{Q C D}$. The values of the $W_{R}$ cross-sections are given in Table 3 .

Since the $W_{R}$ can only decay into two-jets, the mass limits to the $90 \%$ CL is determined for a decay branching ratio of $100 \%$ for $W_{R} \rightarrow \bar{q} q^{\prime}$. In this case the mass of the right handed $\mathrm{W}$ boson is found to be greater than $261 \mathrm{GeV}$ to the $90 \%$ CL. Figure 3 shows the $90 \% \mathrm{CL}$ as a function of $M_{W_{R}}$ and of the branching ratio, normalised to the Standard Model branching ratio.

If the $W_{R}$ can decay to $t \bar{b}$ then its natural width is $\left(M_{W_{R}} / M_{W}\right) \Gamma_{W}$. Since $t \bar{b}$ de- 


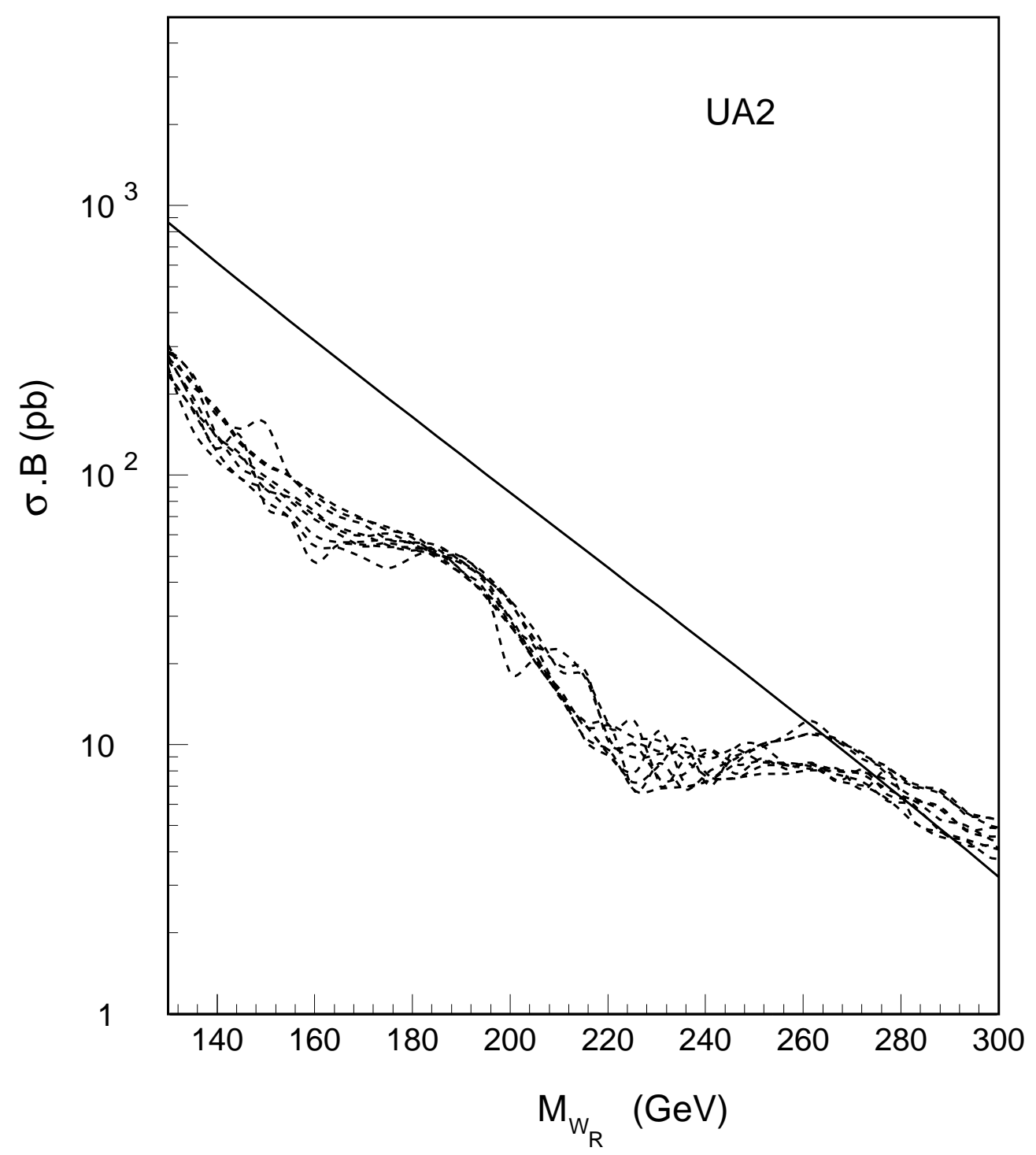

Figure 2: Curves of the $90 \%$ Confidence Limits for $W_{R} \rightarrow \bar{q} q^{\prime}$. The nine dashed lines show the the variations of the limits. The solid line shows the predicted cross section calculated to second order in $\alpha_{s}$ using the structure function set [14] with $\Lambda_{Q C D}=260 \mathrm{MeV}$. 


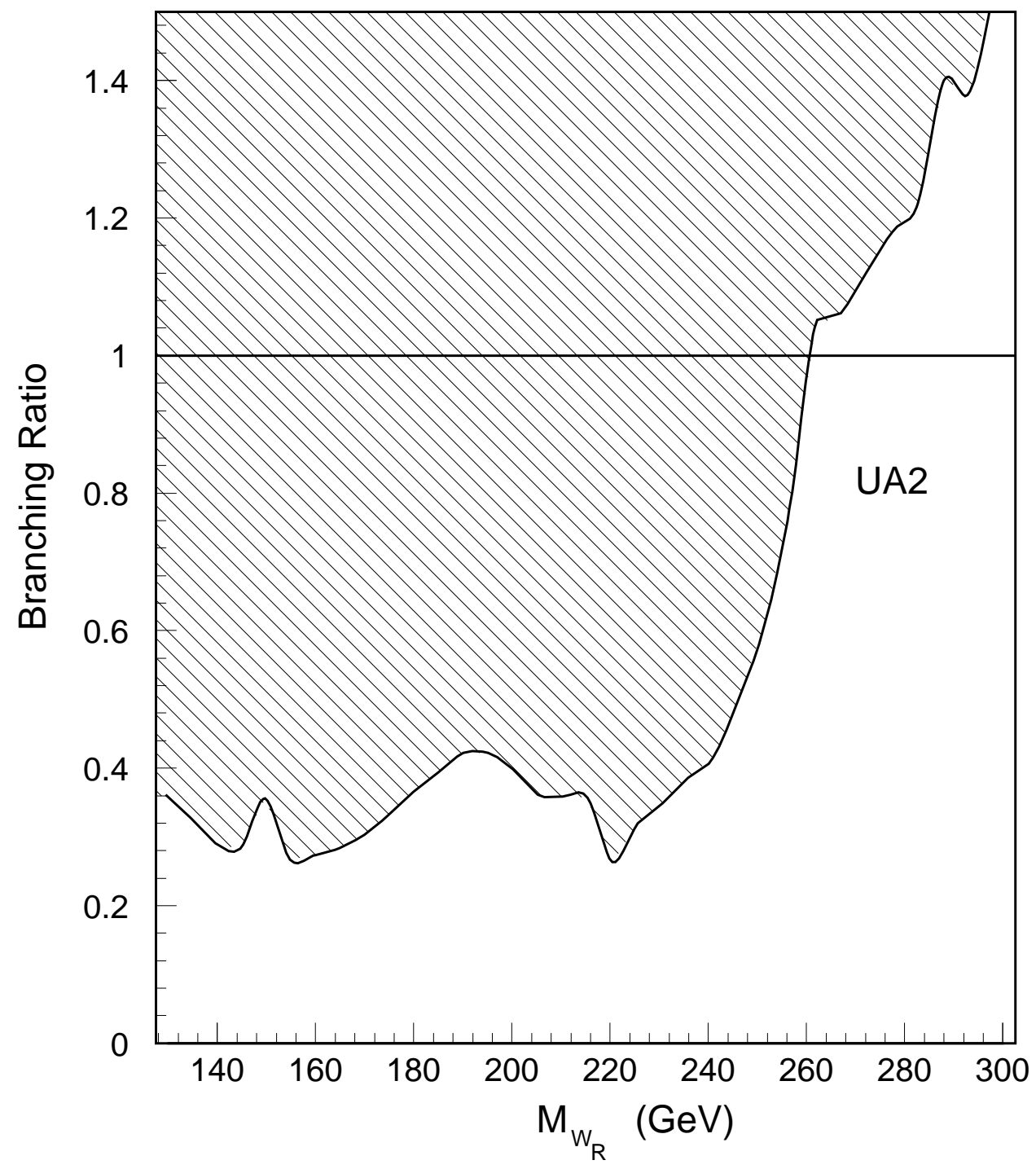

Figure 3: Excluded region to $90 \%$ confidence for $W_{R} \rightarrow \bar{q} q^{\prime}$ assuming that the decay $W_{R} \rightarrow t \bar{b}$ is forbidden (excluded region is hatched). The decay branching ratio is given as a fraction of the Standard Model branching ratio. The solid line shows a branching ratio of 1 for $W_{R} \rightarrow \bar{q} q^{\prime}$. 
Table 3: The $W_{R}$ and $Z^{\prime}$ cross sections as calculated to order $\alpha_{s}{ }^{2}$ with the code developed by [13] using the structure function set [14] with $\Lambda_{Q C D}=260 \mathrm{MeV}$.

\begin{tabular}{|c|c|c|c|c|c|c|c|c|}
\hline $\begin{array}{c}\text { Mass } \\
(\mathrm{GeV})\end{array}$ & $\begin{array}{c}\sigma\left(W_{R}\right) \\
(p b)\end{array}$ & $\begin{array}{c}\sigma\left(Z^{\prime}\right) \\
(p b)\end{array}$ & $\begin{array}{c}\text { Mass } \\
(\mathrm{GeV})\end{array}$ & $\begin{array}{c}\sigma\left(W_{R}\right) \\
(p b)\end{array}$ & $\begin{array}{c}\sigma\left(Z^{\prime}\right) \\
(p b)\end{array}$ & $\begin{array}{c}\text { Mass } \\
(\mathrm{GeV})\end{array}$ & $\begin{array}{c}\sigma\left(W_{R}\right) \\
(p b)\end{array}$ & $\begin{array}{c}\sigma\left(Z^{\prime}\right) \\
(p b)\end{array}$ \\
\hline 100 & 2615.2 & 1344.3 & 170 & 226.2 & 132.4 & 240 & 23.8 & 16.1 \\
110 & 1783.2 & 933.4 & 180 & 163.7 & 97.7 & 250 & 17.2 & 12.0 \\
120 & 1234.0 & 657.7 & 190 & 118.7 & 72.2 & 260 & 12.4 & 8.8 \\
130 & 865.5 & 469.9 & 200 & 86.1 & 53.5 & 270 & 8.9 & 6.5 \\
140 & 612.9 & 338.9 & 210 & 62.5 & 39.7 & 280 & 6.4 & 4.8 \\
150 & 437.4 & 246.4 & 220 & 45.4 & 29.4 & 290 & 4.5 & 3.5 \\
160 & 314.0 & 180.2 & 230 & 32.9 & 21.8 & 300 & 3.2 & 2.6 \\
\hline
\end{tabular}

cays do not contribute to the two-jet decay channel (defined by the algorithm used in this analysis), the branching ratio of $W_{R}$ to two-jets is $\frac{2}{3}$, giving $M_{W_{R}}>251 \mathrm{GeV}$ at the $90 \%$ CL. The $90 \% \mathrm{CL}$ are shown in Fig 4 as a function of $M_{W_{R}}$ and decay branching ratio.

\subsection{Mass Limits of an Additional Standard Model Z Boson}

If an additional $\mathrm{Z}$ boson with couplings identical to the Standard Model exists with a natural width given by the width of the $Z$ boson scaled to the $Z^{\prime}$ mass,

$$
\Gamma_{Z^{\prime}}=\left(\frac{M_{Z^{\prime}}}{M_{Z}}\right) \Gamma_{Z} .
$$

The values of $M_{Z}$ and $\Gamma_{Z}$ used in this analysis are the ones quoted in Ref [12]. After applying a mass correction similar to the one given in $\mathrm{Eq}(9)$ the $90 \%$ confidence region is calculated in the same way as for the $W_{R}$. The $90 \% \mathrm{CL}$ are shown in Fig 5 as a function of $M_{Z^{\prime}}$ and decay branching ratio. If a branching ratio for $Z^{\prime} \rightarrow \bar{q} q$ of $100 \%$ is assumed a mass limit of $252 \mathrm{GeV}$ is obtained at the $90 \% \mathrm{CL}$. If the Standard Model branching ratio of $70 \%$ is assumed the mass limit becomes $237 \mathrm{GeV}$ at the $90 \% \mathrm{CL}$, but the limit is sensitive to the choice of branching ratio in the region $180<M_{Z^{\prime}}<200 \mathrm{GeV}$ (see Fig 5).

\subsection{Mass Limits of Excited Quark States.}

Mass limits have been determined for excited quarks decaying into two jets using the model described in Ref [15].

Since the production processes of excited quarks are different from those of vector bosons, the efficiencies, decay widths and the detector mass responses must be 


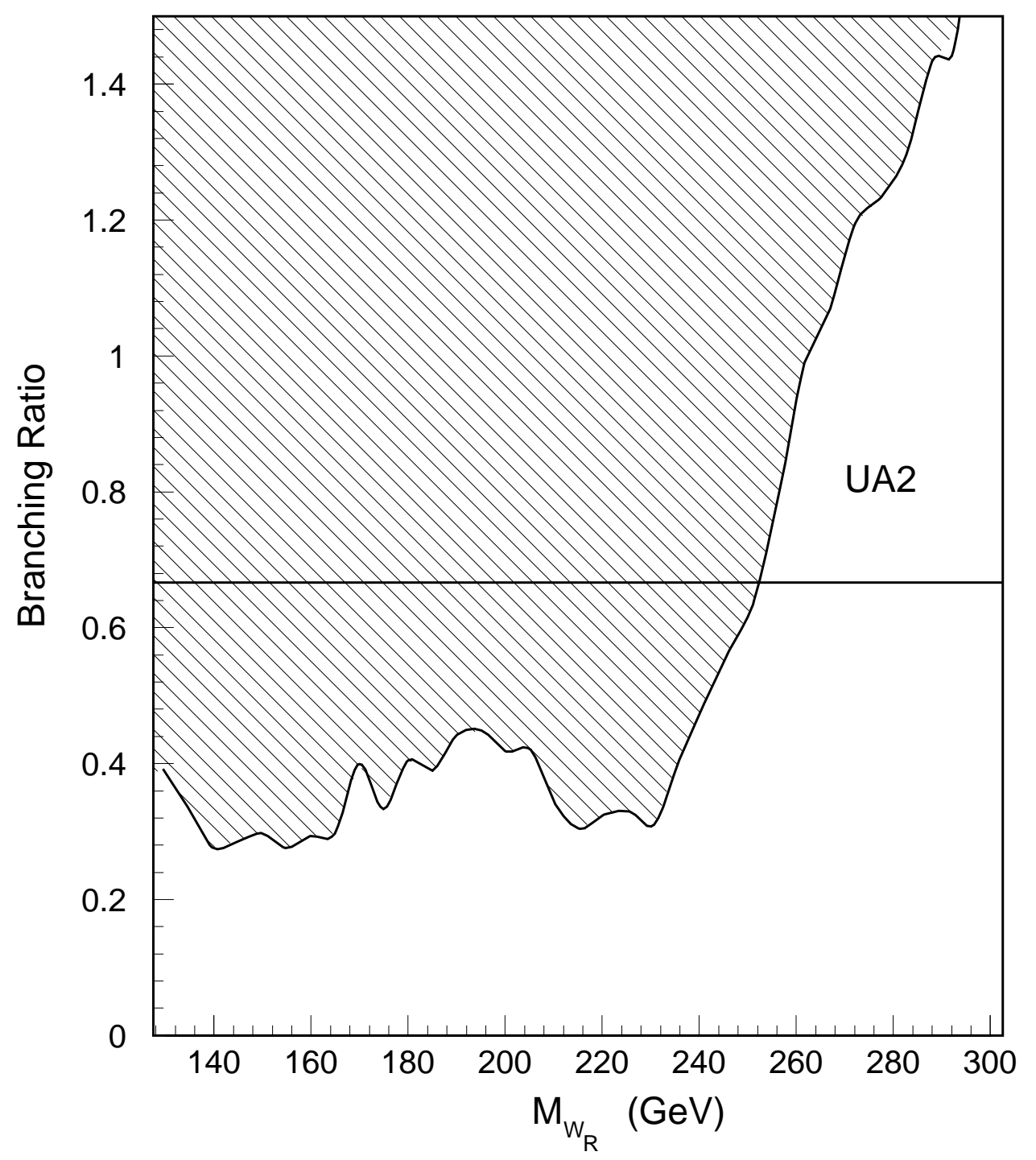

Figure 4: Excluded region to $90 \%$ confidence for $W_{R} \rightarrow \bar{q} q^{\prime}$ assuming that the decay $W_{R} \rightarrow t \bar{b}$ is allowed (excluded region is hatched). The branching ratio is given as a fraction of the Standard Model branching ratio. The solid line shows a branching ratio of $\frac{2}{3}$ for $W_{R} \rightarrow \bar{q} q^{\prime}$. 


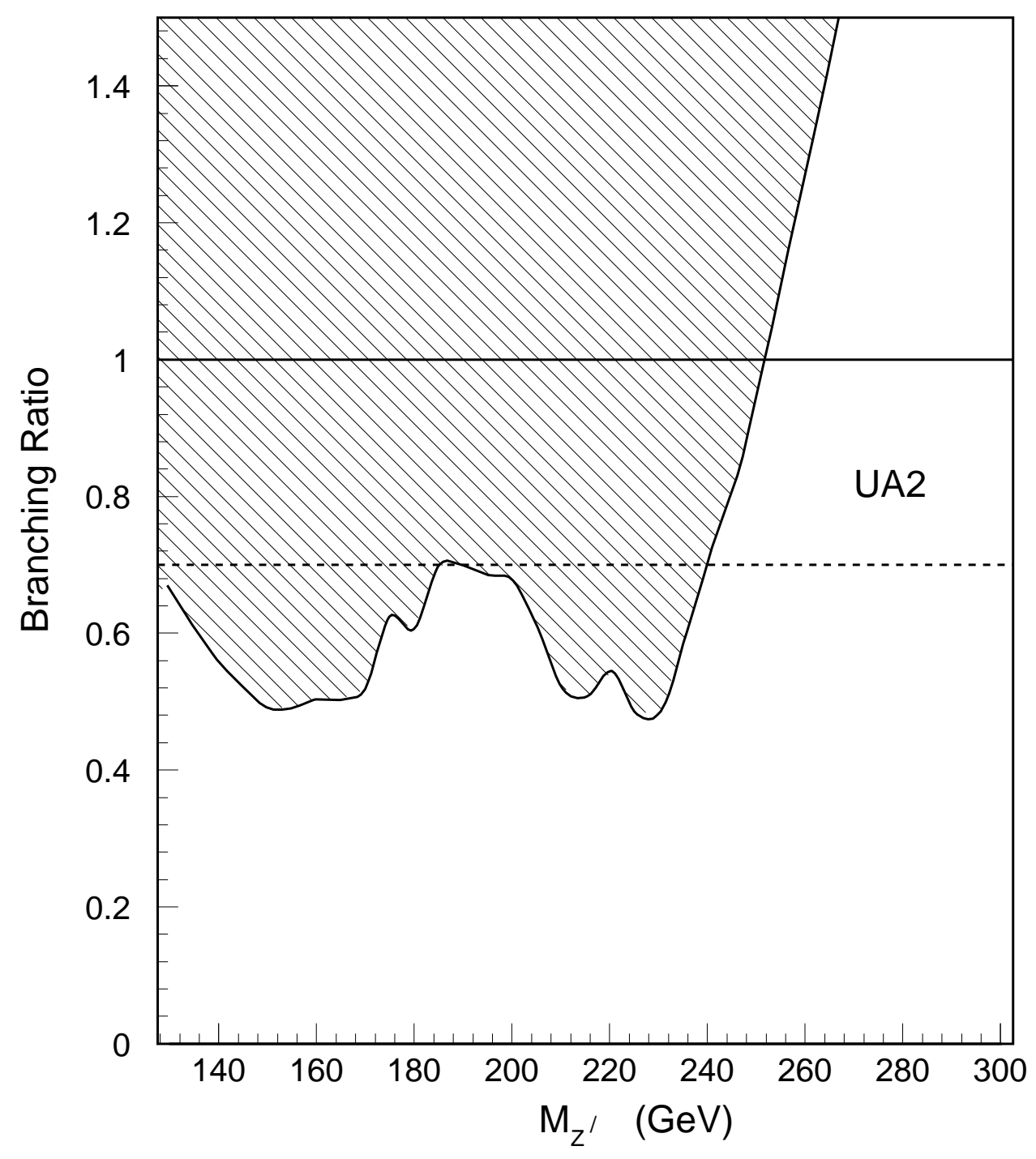

Figure 5: Excluded region to $90 \%$ for $Z^{\prime} \rightarrow \bar{q} q$, (excluded region is hatched). The branching ratio is given as a fraction of Standard Model branching ratio. The solid line shows a branching ratio of 1 for $Z^{\prime} \rightarrow \bar{q} q$ whilst the dashed line shows a branching ratio of 0.7 . 
recalculated. This is done by producing a mixture of $u^{*}$ and $d^{*}$ events using a Monte Carlo Program [8] and repeating the studies described in section 5.2 for $Z^{\prime}$ events. The results of these calculations are given in table 4 . The errors are larger than those calculated for the $Z^{\prime}$ events because only 3,000 excited quark events were generated at each mass compared to $10,000 Z^{\prime}$ events.

Table 4: Mass Response, R, $\sigma$ and efficiency of the fitting procedure for excited quarks.

\begin{tabular}{|c|c|c|c|c|c|}
\hline $\begin{array}{c}M_{X} \\
(\mathrm{GeV})\end{array}$ & $\begin{array}{c}\Gamma_{X} \\
(\mathrm{GeV})\end{array}$ & $\begin{array}{c}\mathrm{R} \\
\left(M_{J J} / M_{X}\right)\end{array}$ & $\begin{array}{c}\sigma \\
(\%)\end{array}$ & $\begin{array}{c}\epsilon_{\text {fit }} \\
(\%)\end{array}$ & $\begin{array}{c}\epsilon_{A L L} \\
(\%)\end{array}$ \\
\hline 150 & 5.9 & $0.90 \pm 0.03$ & $11.2 \pm 2.1$ & $82.9 \pm 21.9$ & $21.9 \pm 5.2$ \\
200 & 8.0 & $0.907 \pm 0.008$ & $9.2 \pm 1.0$ & $81.8 \pm 36.5$ & $23.7 \pm 10.7$ \\
250 & 10.0 & $0.924 \pm 0.005$ & $9.0 \pm 1.4$ & $71.2 \pm 25.6$ & $23.7 \pm 8.7$ \\
300 & 11.9 & $0.913 \pm 0.004$ & $8.2 \pm 0.3$ & $72.2 \pm 27.0$ & $23.9 \pm 9.1$ \\
\hline
\end{tabular}

The efficiencies for detecting excited quarks decaying to two-jets are systematically higher than the efficiencies for detecting two-jet decays of additional vector bosons (see Tables 2 and 4 ). This is caused by the differences between the line shape of the excited quark (see Fig 6 ) and the line shape of $Z^{\prime}$ two-jet decays. The number of events in the low mass tail is greatly reduced since the excited quarks can only decay into their unexcited counterparts. Only jets caused by the production of gluons, up quarks and down quarks are produced by the decays of $u^{*}$ and $d^{*}$ quarks whilst vector bosons also decay to heavy quarks which in turn decay into leptons and neutrinos leading to a low-mass tail.

The production and decay via gauge interactions only is the simplest possible model describing the production of excited quarks. The natural width of the excited quark is given by the sum:

$$
\Gamma_{q^{*}}=\Gamma\left(q^{*} \rightarrow q \gamma\right)+\Gamma\left(q^{*} \rightarrow q Z\right)+\Gamma\left(q^{*} \rightarrow q^{\prime} W\right)+\Gamma\left(q^{*} \rightarrow q g\right)
$$

where the partial widths are calculated using the formulae in Ref [15] with the following parameter values $f_{s}=f=f^{\prime}=1, m^{*}=\Lambda_{C}, \Lambda_{Q C D}^{(4)}=260 \mathrm{MeV}$.

Using the above value of $\Gamma_{q^{*}}$ the $90 \% \mathrm{CL}$ on the production cross-section of excited quarks are determined in the same way as for the $W_{R}$ and the $Z^{\prime}$. The mass correction is then applied using the mass response for excited quarks and the $90 \%$ confidence region is determined by comparing the $90 \%$ CL with the production cross-sections of $u^{*}$ and $d^{*}$ predicted by theory (see Table 5 ).

Three cases are considered depending on the masses of the excited quarks. For the first case, $M_{u^{*}}=M_{d^{*}}$, and where the mass peaks of $u^{*}$ and $d^{*}$ are indistinguishable. The $90 \%$ confidence region is then calculated using the combined $u^{*}$ 


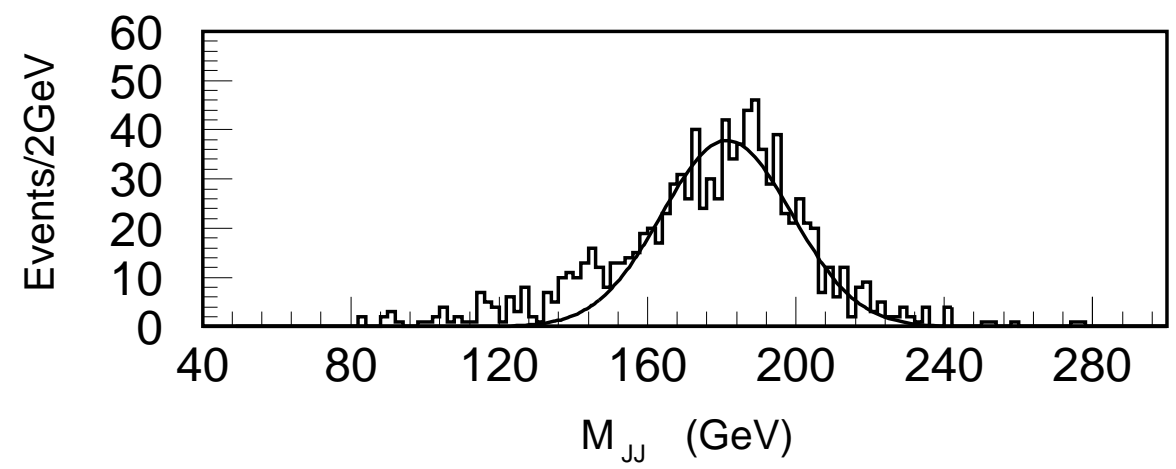

Figure 6: Monte Carlo generated $200 \mathrm{GeV} u^{*}+d^{*}$ decaying to two-jets. The curve shows the best fit to the signal.

Table 5: Excited quark production cross sections as calculated to the Born level using the structure function set [14] with $\Lambda_{Q C D}=260 \mathrm{MeV}$.

\begin{tabular}{|c|c|c|c|c|c|c|c|}
\hline $\begin{array}{c}\text { Mass } \\
(\mathrm{GeV})\end{array}$ & $\begin{array}{c}\sigma\left(u^{*}+d^{*}\right) \\
(p b)\end{array}$ & $\begin{array}{c}\sigma\left(u^{*}\right) \\
(p b)\end{array}$ & $\begin{array}{c}\sigma\left(d^{*}\right) \\
(p b)\end{array}$ & $\begin{array}{c}\text { Mass } \\
(\mathrm{GeV})\end{array}$ & $\begin{array}{c}\sigma\left(u^{*}+d^{*}\right) \\
(p b)\end{array}$ & $\begin{array}{c}\sigma\left(u^{*}\right) \\
(p b)\end{array}$ & $\begin{array}{c}\sigma\left(d^{*}\right) \\
(p b)\end{array}$ \\
\hline 120 & 8673.2 & 6172.2 & 2500.9 & 220 & 97.9 & 75.3 & 22.6 \\
130 & 5321.8 & 3821.9 & 1499.9 & 230 & 64.2 & 49.6 & 14.5 \\
140 & 3290.6 & 2383.8 & 906.7 & 240 & 42.6 & 33.2 & 9.5 \\
150 & 2058.2 & 1503.6 & 554.6 & 250 & 28.1 & 22.0 & 6.1 \\
160 & 1299.3 & 956.7 & 342.6 & 260 & 18.8 & 14.7 & 4.0 \\
170 & 829.5 & 615.8 & 213.8 & 270 & 12.5 & 9.9 & 2.7 \\
180 & 530.7 & 397.1 & 133.6 & 280 & 8.4 & 6.6 & 1.8 \\
190 & 348.1 & 262.3 & 85.7 & 290 & 5.6 & 4.4 & 1.2 \\
200 & 225.2 & 170.9 & 54.3 & 300 & 3.8 & 3.0 & 0.8 \\
210 & 148.1 & 113.2 & 34.9 & & & & \\
\hline
\end{tabular}


and $d^{*}$ production cross-sections and is shown in Fig 7 . The branching ratio of $q^{*} \rightarrow$ two-jets is given by $\Gamma\left(q^{*} \rightarrow q g\right) / \Gamma_{q^{*}}$. The mass limit for combined $u^{*}$ and $d^{*}$ production is $M_{q^{*}}>288 \mathrm{GeV}$.

The two other cases examine the situation where $M_{u^{*}} \neq M_{d^{*}}$. If one considers only $u^{*}$ production assuming $M_{d^{*}} \gg M_{u^{*}}$, then the $90 \%$ confidence region is given in Fig 8a and the resulting mass limit is $M_{u^{*}}>277 \mathrm{GeV}$. The mass limit for $d^{*}$ production assuming $M_{u^{*}} \gg M_{d^{*}}$ is $M_{d^{*}}>247 \mathrm{GeV}$ (see Fig 8b). The reason for the lower mass limit for $d^{*}$ production is that the fraction of up quarks in the proton is approximately twice that of down quarks in protons and hence the production cross section of $d^{*}$ is smaller than that of $u^{*}$ quarks (see Table 5).

It must be noted that no $\mathrm{K}$-factors are included in the theoretical calculations of the excited quark cross-sections. Since these K-factors allow for higher order contributions to the cross-section the $90 \% \mathrm{CL}$ are conservative. A correction for these higher order effects can be made by adjusting the branching ratio by a $\mathrm{K}$-factor and reading off the mass limit from Figs 7 and 8 .

\section{Conclusion}

This paper has established limits on the production of additional heavy vector bosons decaying into two jets. The mass of $W_{R}$ is found to be greater than $261 \mathrm{GeV}$ at the $90 \%$ CL with standard model couplings if decays to top quarks are forbidden. If decays to top quarks are allowed then the $W_{R}$ mass bound becomes $251 \mathrm{GeV}$. The mass of an additional $Z$ boson, $Z^{\prime}$, is found to be greater than $252 \mathrm{GeV}$ at the $90 \%$ CL. Limits have also been established on the production of excited quarks decaying into two jets via gauge interactions. The masses of degenerate $u^{*}$ and $d^{*}$ quarks is found to be greater than $288 \mathrm{GeV}$ at the $90 \% \mathrm{CL}$. For the non-degenerate cases, the $90 \% \mathrm{CL}$ are $M_{u^{*}}>277 \mathrm{GeV}$ if $M_{d^{*}} \gg M_{u^{*}}$; and $M_{d^{*}}>247 \mathrm{GeV}$ if $M_{u^{*}} \gg M_{d^{*}}$

\section{Acknowledgements}

The technical staff of the institutes collaborating in UA2 have contributed substantially to the construction and operation of the experiment. We deeply thank them for their support.

The experiment would not have been possible without the very successful operation of the improved CERN $\bar{p} p$ Collider, whose staff and coordinators we sincerely thank for their collective effort.

Financial support from the Schweizerischen Nationalfonds zur Förderung der Wissenschaftlichen Forschung to the Bern group, from the UK Science and Engineering Research Council to the Cambridge group, from the Bundesministerium 


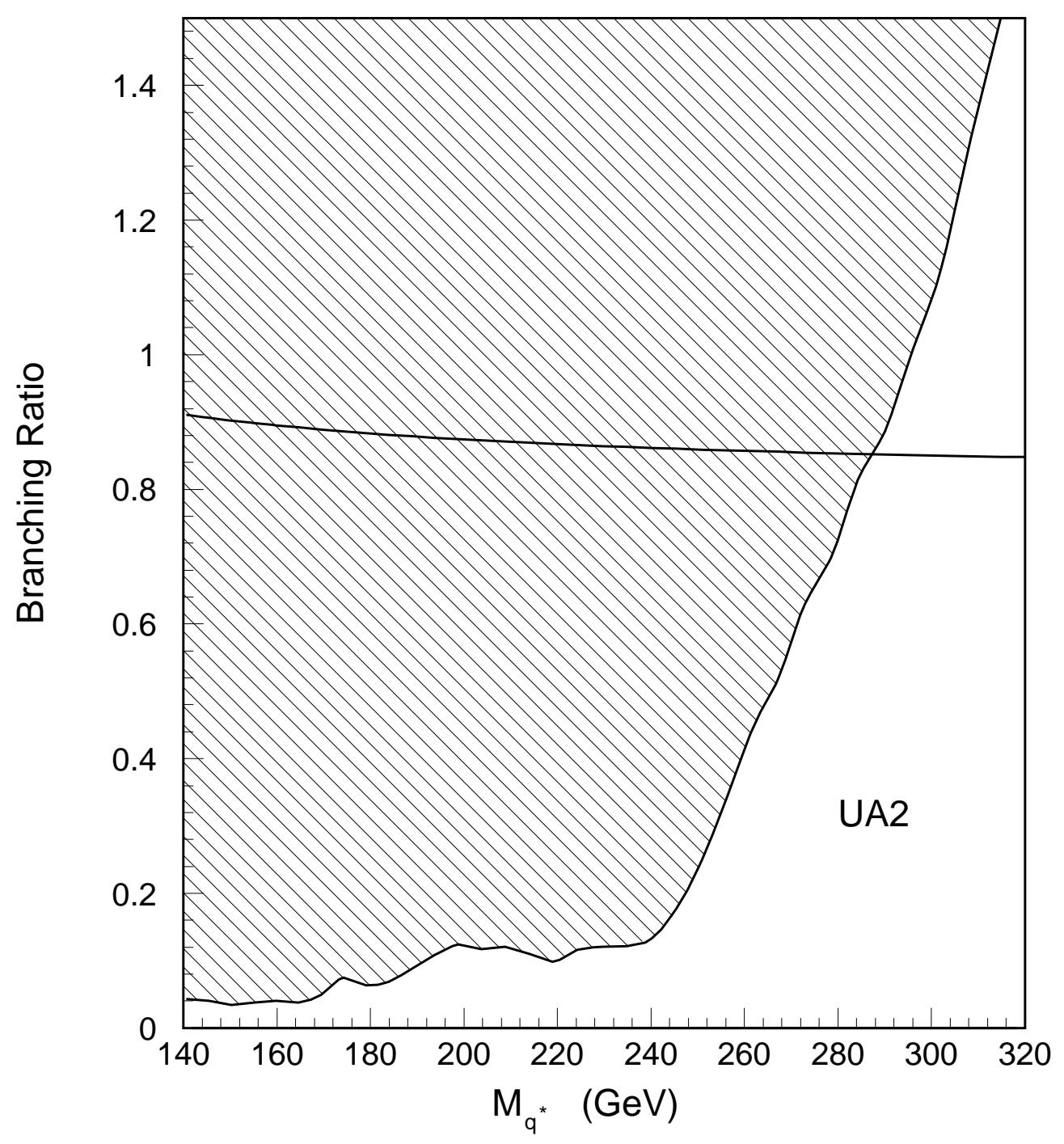

Figure 7: 90\% Confidence region for $u^{*}+d^{*} \rightarrow q g$ (excluded region is hatched). The solid line shows the branching ratio for $u^{*}$ and $d^{*}$ decays to two-jets. 

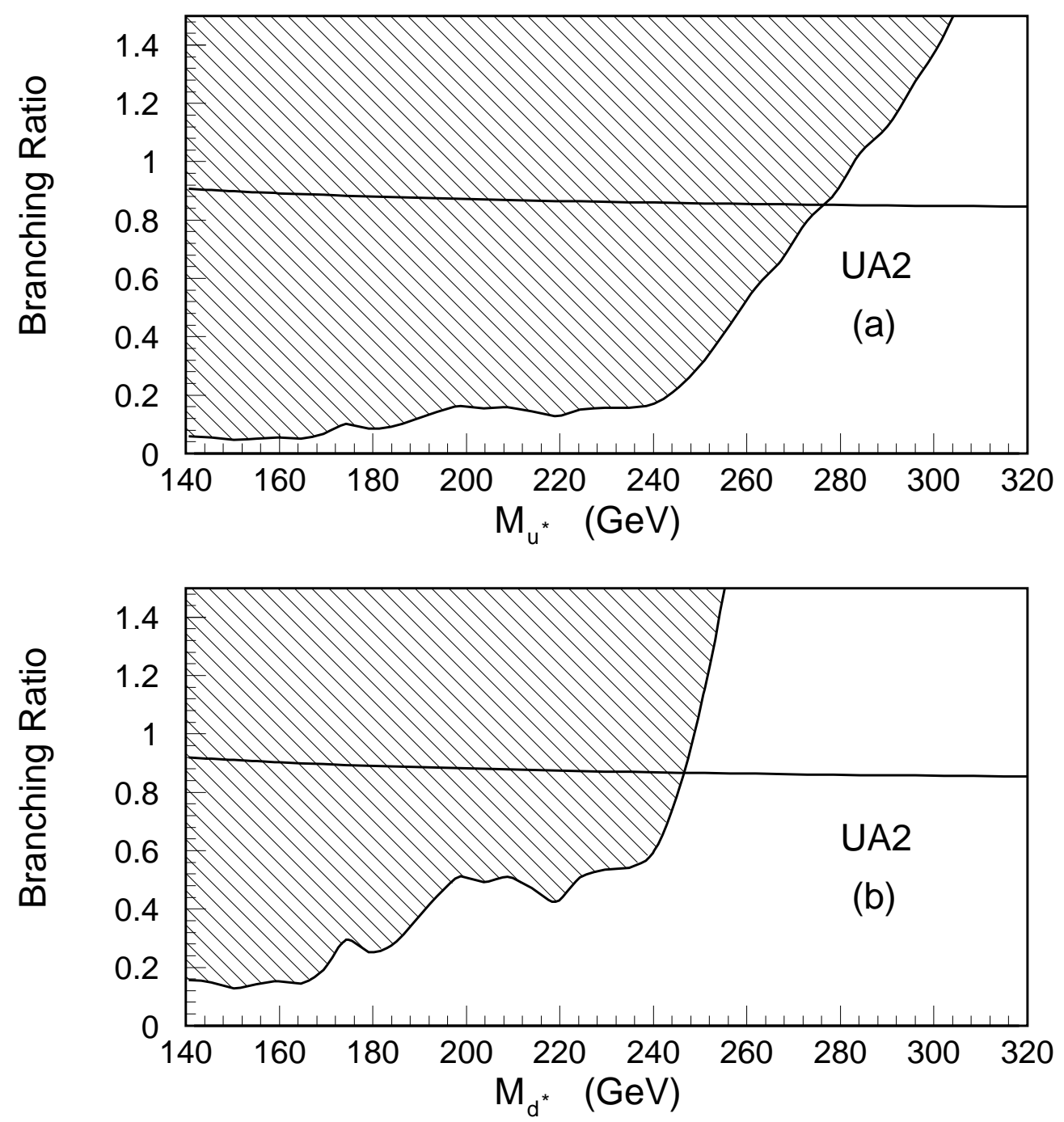

Figure 8: $90 \%$ Confidence region for $q^{*} \rightarrow q g$ (excluded region is hatched). Figure a) gives the $90 \%$ Confidence region for $u^{*} \rightarrow u g$ whilst b) gives the $90 \%$ Confidence region for $d^{*} \rightarrow d g$. In both cases the branching ratio to two-jets is given by the solid line. 
für Forschung und Technologie to the Dortmund and Heidelberg groups, from the Australian Research Council, the CRA Pty Ltd, and the Victorian Education Foundation to the Melbourne group, from the Institut National de Physique Nucléaire et de Physique des Particules to the Orsay group, from the Istituto Nazionale di Fisica Nucleare to the Milano, Pavia, Perugia and Pisa groups and from the Institut de Recherche Fondamentale (CEA) to the Saclay group are acknowledged.

\section{References}

[1] CDF Collaboration, F. Abe et al., Phys. Rev. Lett. 67 (1991) 2609 and 68 (1992) 1463.

[2] UA2 Collaboration, M. Banner et al., Phys. Lett. B118 (1982) 203;

UA1 Collaboration, G. Arnison et al., Phys. Lett. B123 (1983) 115.

[3] For a review see: P. Bagnaia and S. D. Ellis, Ann. Rev. Nucl. Part. Sc. 38 (1988) 659.

[4] UA2 Collaboration, J. Alitti et al., Z. Phys. C 49 (1991) 17.

[5] UA2 Collaboration, CERN SPSC 84-30, 84-95 (1984) and 85-3 (1985), (unpublished);

UA2 Collaboration, C.N. Booth, Proceedings 6th Topical Workshop on ProtonAntiproton Collider Physics, Aachen, 1986, eds. K. Eggert et al., (World Scientific, Singapore, 1987) 381.

[6] A. Beer et al., Nucl. Inst. Meth. 224 (1984) 360.

[7] T. Koch, Aufbau und Test eines Flugzeitsystems, HD-IHEP 89-05, Heidelberg (1989) (unpublished).

[8] H.-U. Bengtsson and T. Sjöstrand, PYTHIA 4.8, Comput. Phys. Commun. 46 (1987) 43.

[9] T. Sjöstrand and M. Bengtsson, JETSET 6.9, Comput. Phys. Commun. 43 (1987) 367.

[10] UA2 Collaboration, J. Alitti et al., Phys. Lett. B276 (1992) 354.

[11] UA2 Collaboration, J. Alitti et al., Phys. Lett. B276 (1992) 365.

[12] Particle Data group, Review of Particle Properties, Phys. Rev. D 45-II (1992).

[13] R. Hamberg, W.L. van Neerven, and T. Maatsura, Nucl. Phys. B359 (1991) 343.

[14] M. Diemoz, F. Ferroni, E. Longo and G. Martinelli, Z. Phys. C 39 (1988) 21.

[15] U. Baur, M. Spira and P.M. Zerwas, Phys. Rev. D 42 (1990) 815. 